\title{
Integrated Optical Addressing of a Trapped Ytterbium Ion
}

\author{
M. Ivory®, 'W. J. Setzer®, N. Karl®, H. McGuinness, C. DeRose, M. Blain, D. Stick®, M. Gehl, and L. P. Parazzoli® \\ Sandia National Laboratories, Albuquerque, New Mexico 87185, USA
}

(Received 19 November 2020; revised 27 April 2021; accepted 31 August 2021; published 16 November 2021)

\begin{abstract}
We report on the characterization of heating rates and photoinduced electric charging on a microfabricated surface ion trap with integrated waveguides. Microfabricated surface ion traps have received considerable attention as a quantum information platform due to their scalability and manufacturability. Here, we characterize the delivery of 435-nm light through waveguides and diffractive couplers to a single ytterbium ion in a compact trap. We measure an axial heating rate at room temperature of $0.78 \pm 0.05 \mathrm{q} / \mathrm{ms}$ and see no increase due to the presence of the waveguide. Furthermore, the electric field due to charging of the exposed dielectric outcoupler settles under normal operation after an initial shift. The frequency instability after settling is measured to be $0.9 \mathrm{kHz}$.
\end{abstract}

DOI: 10.1103/PhysRevX.11.041033

Subject Areas: Atomic and Molecular Physics,

Quantum Physics, Quantum Information

\section{INTRODUCTION}

Though quantum information applications have motivated most of the development of microfabricated surface ion traps [1,2], many of the same advantages and challenges are also applicable to atomic clocks. This idea is particularly true for those using optical transitions, which require atomic confinement within the Lamb Dicke regime. While neutral atoms achieve this confinement in an optical lattice with many atoms, a conventional-sized ion trap is limited to small numbers of crystallized ions which limit the signal-to-noise ratio. Despite this limitation, an ion atomic clock using a single ion in a macroscale trap has achieved unprecedented fractional systematic uncertainties better than $1 \times 10^{-18}$ using quantum logic spectroscopy, albeit in a laboratory scale environment $[3,4]$.

Furthermore, microfabricated ion traps have been extended to support many independently addressable individual trapping sites. This capability maintains high performance in a deployable configuration that combines low size, weight, and power (SWaP). Additional ions can also be used to replace lost ions, monitor environmental conditions, eliminate Dicke effect errors using staggered optical interrogation sites, and optimize feedback to the local oscillator using different interrogation times to further extend the coherence time. This latter benefit fundamentally improves the clock stability scaling [5]. Finally, the

\footnotetext{
*mkivory@sandia.gov
}

Published by the American Physical Society under the terms of the Creative Commons Attribution 4.0 International license. Further distribution of this work must maintain attribution to the author(s) and the published article's title, journal citation, and DOI. ability to integrate waveguides, detectors, and other photonic elements into the surface trap adds scalability and robustness compared to the alternative of free-space optics [6-9].

The benefits provided by microfabricated traps have led to significant advances, but several challenges remain, both in the microfabricated trap integration and in understanding the added systematic effects on clock operation due to the integrated elements. First, optical transitions of many trapped-ion species lie in the blue to ultraviolet spectrum where transmission through standard silicon nitride waveguides is inefficient. Instead, one must use aluminum nitride or alumina to achieve low loss $[10,11]$. To date, there has been one demonstration of trapped-ion manipulation using waveguide delivery of blue light, which included the photoionization and Doppler cooling wavelengths for $\mathrm{Sr}^{+}$at $405 \mathrm{~nm}, 461 \mathrm{~nm}$, and $422 \mathrm{~nm}$ [12].

A second outstanding challenge for microfabricated traps is anomalous heating, which couples noise to the motional state of the ion. Previous studies [13-16] have found that the heating rate dependence of a trapped-ion scales with the ion-electrode distance approximately as $\mathrm{d}^{-4}$. Furthermore, this heating rate is expected to increase considerably over a dielectric as opposed to a metallic layer [17], which is a potential complication to photonics integration that is fabricated with dielectric materials.

Finally, the presence of light on a dielectric material creates electrostatic fields through photoinduced charging. This charging impacts the performance and operation of the clock by moving the ion relative to the optical beams, increasing micromotion, and creating systematic shifts in the transition frequency.

In this work, we study the electric field and the heating rate impact on an ion in proximity to an integrated photonic 
outcoupler that is used to deliver light at $435 \mathrm{~nm}$ to a trapped ${ }^{171} \mathrm{Yb}^{+}$ion $20 \mu \mathrm{m}$ above the trap surface (Fig. 1). This ion height is the lowest published to date. Ion heights in surface traps are a concern because of the correlation of the heating rate of the ion to its height above the trap surface. Despite the increase in ion heating, lower heights are desirable, particularly in architectures in which each ion is confined in an individual trapping site or multiple trapping sites are required on a single chip, such as the QCCD architecture $[18,19]$. In these cases, the lower ion height allows for closer ion spacing due to the smaller geometric size of the dc trapping electrodes, and thus more ions can be stored on a single chip. Additionally, the lower ion height also reduces the width of the rf electrode, which in turn reduces the overall capacitance and thus rf power dissipation of the trap chip - an advantage in SWaP critical applications such as a deployable optical atomic clock.

The trap includes integrated waveguides that connect diffractive grating input and output couplers below the top metal level; using the integrated photonics, we have demonstrated hyperfine spectroscopy, ground-state motional sideband cooling, and heating rate measurements. We compare heating rates over a range of $80 \mu \mathrm{m}$ along the trap to measure the impact of an exposed dielectric on the ion. We measure heating rates of $0.78 \pm 0.05 \mathrm{q} / \mathrm{ms}$, which agree with previously reported data for larger ion-surface distances following a d ${ }^{-4}$ scaling. Surprisingly, we see no increase in the heating rates as the ion approaches the dielectric grating, which gives encouraging evidence that non-CMOS compatible fabrication steps such as indium tin oxide (ITO) coatings may not be necessary to achieve adequate heating rates in photonics-integrated chips. However, we observe photoinduced charging of the dielectric, which leads to a shift in the measured trap frequency.

\section{TRAP DESIGN AND FABRICATION}

The trap used in this work was designed and fabricated at Sandia National Laboratories using CMOS compatible silicon MEMS fabrication technologies in a configuration similar to those previously reported [20]. Images of the trap are shown in Fig. 1. Multiple metal layers are used to route rf and dc signals to electrodes on the top metal level. The top metal layer, containing the rf routing and trap dc control electrodes, overhangs the underlying $\mathrm{SiO}_{2}$ dielectric layer to eliminate a direct line of sight between the ion and dielectric. Notable to this particular trap is the integration of silicon nitride waveguides embedded in the $\mathrm{SiO}_{2}$ intermetal dielectric and routed between metal levels and around vertical electrical vias made of chemical-vapor-deposited tungsten for delivery of 435-nm light. Light is coupled in and out of the waveguide using diffractive gratings. Figure 1(c) shows the trapping region with a loading hole on the left and four output gratings to its right. Figure 1(a) shows a zoom-in of the loading hole site, and Fig. 1(b) shows a zoom-in of the leftmost output grating site. The
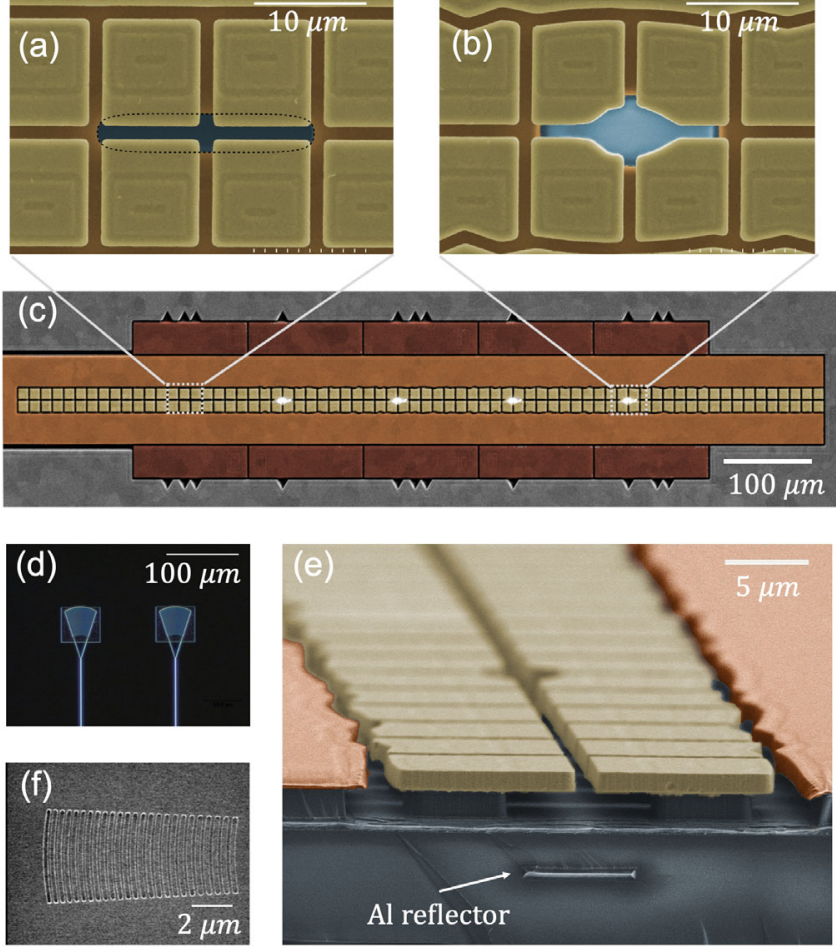

FIG. 1. (a) False-colored scanning electron micrograph (SEM) image of the loading hole region of the trap and dc control electrodes (yellow) in the topmost metal layer. The dark gray dotted line indicates cutout beneath the overhanging metal layer to allow backside loading. (b) False-colored SEM image of the region above the leftmost output diffraction grating, shown in blue beneath the overhanging metal layer. (c) False-colored SEM image of the trapping region of the surface trap showing dc control electrodes (yellow), rf electrodes (orange), and dc bias electrodes (brown). Triangles above and below dc bias electrodes are for beam alignment. The trap region shows the loading hole on the left and four output diffraction gratings. (d) Dark field optical micrograph image of the input diffraction gratings for coupling light to the waveguides. (e) False-colored SEM imaged sectional view of the trap showing the overhanging top metal electrodes and $\mathrm{Al}$ reflector below an output grating. (f) SEM image of a diffractive output coupler etched in $\mathrm{SiN}_{x}$ for coupling light from the waveguides and focusing light $20 \mu \mathrm{m}$ above the chip surface.

small electrodes immediately above and below the trap axis are dc control electrodes. The rf electrodes are the long rails between the dc control electrodes and the outer dc bias electrodes.

Plasma-enhanced chemical-vapor-deposition (PECVD) silicon nitride waveguides with a thickness of $70 \mathrm{~nm}$ are deposited within the 2- $\mu \mathrm{m} \mathrm{SiO}_{2}$ dielectric layer between trap metal layers; the silicon nitride layer is kept thin to reduce material absorption. We observe a propagation loss of $-2.5 \mathrm{~dB} / \mathrm{cm}$ at $435 \mathrm{~nm}$ in test waveguides fabricated with similar processes. The input grating [Fig. 1(d)] is designed for free-space coupling with a relatively large beam waist of $15 \mu \mathrm{m}$. The output grating [Fig. 1(f)] is 
located between the dc electrodes and designed to produce a beam that focuses at the height of the ion. Utilizing the metal trap routing layer, an $\mathrm{Al}$ reflector is positioned below the output grating to increase efficiency [Fig. 1(e)].

Simulations of the ideal structure show $-6.5 \mathrm{~dB}$ input coupling loss and $-1.6 \mathrm{~dB}$ output coupling loss for an expected total through-chip insertion loss of approximately $-9.7 \mathrm{~dB}$. The output beam is simulated to emit at a $63^{\circ}$ angle with respect to the plane of the chip and have a focused beam waist of $1.45 \mu \mathrm{m}$ at the height of the ion, with expected polarization parallel to the chip surface. In a separate die external to the ultrahigh vacuum-chamber trapped-ion system, we measure a through-chip insertion loss of $-22 \mathrm{~dB}$ and a beam waist of $2.5 \mu \mathrm{m}$ at a height of $20 \mu \mathrm{m}$ above the chip. The difference in the beam waists is likely due to fabrication tolerances, while the difference in through-chip insertion loss is likely due to reduced input coupling from a cleaved fiber. In future devices, we plan to use edge coupling instead of the input diffraction grating, which is expected to reduce input coupling loss to $-3 \mathrm{~dB}$ with a photolithography process and to $-0.5 \mathrm{~dB}$ with ebeam lithography.

\section{EXPERIMENTAL DETAILS AND RESULTS}

The ${ }^{171} \mathrm{Yb}^{+}$ions are produced via an isotope-selective twophoton ionization process [21], Doppler cooled to an average number of quanta $\langle n\rangle \approx 3$, and sideband cooled to achieve $\langle n\rangle$ as low as 0.1 quanta. A rf voltage at $74.5 \mathrm{MHz}$ is applied to the rf rails (yellow in Fig. 1), and quasistatic voltages are applied to electrodes to control the axial position and cancel stray fields. Typical secular axial trap frequencies are tunable between $2 \pi \times 2.5$ and $2 \pi \times 5.2 \mathrm{MHz}$, and radial trap frequencies are $2 \pi \times 12.7 \mathrm{MHz}$. These frequencies are verified via spectroscopy on the $\left|2 S_{1 / 2}, F=0\right\rangle$ to $\left|2 D_{3 / 2}, F=2\right\rangle$ quadrupole clock transition. All beams are delivered via free-space optics with the exception of the 435$\mathrm{nm}$ beam, which addresses the quadrupole transition and can be switched between free-space and waveguide delivery.

\section{A. Heating rates}

We measure the motional heating rate $\langle\dot{n}\rangle$, or the time rate of change of the average number of quanta $n$ for a given motional mode, for the axial trap mode on the $\left|2 S_{1 / 2}, F=0, m_{F}=0\right\rangle$ to $\left|2 D_{3 / 2}, F=2, m_{F}=+2\right\rangle$ transition by comparing the amplitudes of the red and blue axial sidebands [22]. The $\Delta m_{F}=+2$ transition was chosen because it has the strongest coupling with the free-space beam for our geometry.

Results of a heating rate measurement taken with the sideband asymmetry method can be seen in Fig. 2. After sideband cooling and without any additional heating time, we achieve $\langle n\rangle \sim 0.1$, and the red axial sideband is significantly suppressed. After a heating time of $3 \mathrm{~ms}$, the ion reaches a temperature roughly the same as the

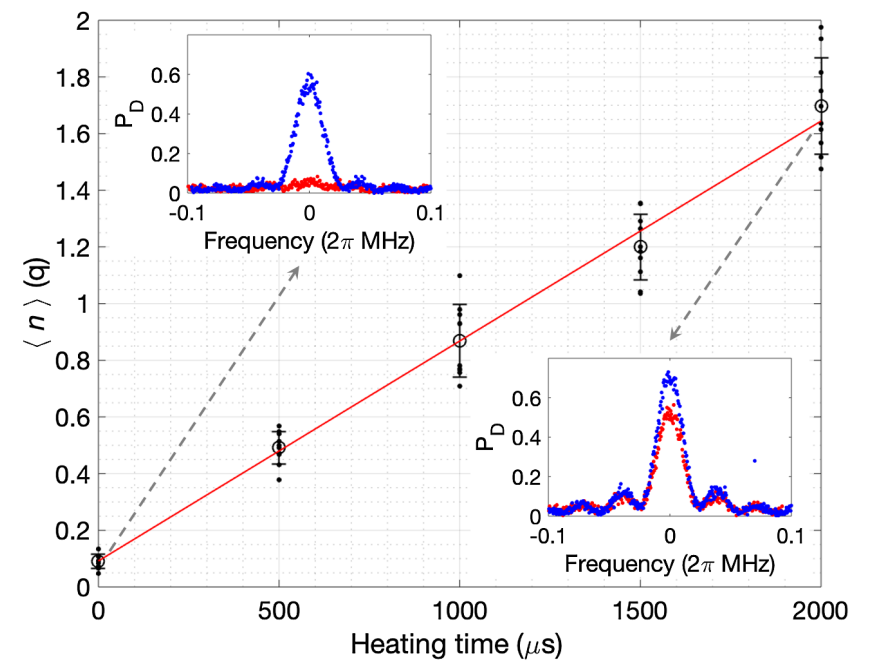

FIG. 2. $\langle n\rangle$ as a function of time using sideband amplitudes over the loading hole. Insets show the corresponding sideband amplitude measurements for $\langle n\rangle=0.1$ (top) and $\langle n\rangle=3$ (bottom). These measurements were taken using a free-space clock beam. The red and blue data correspond to the red and blue sidebands.

Doppler cooling temperature, or $\langle n\rangle \sim 3$ quanta, so we limit our heating time in a typical measurement to $2 \mathrm{~ms}$ or less, as shown in Fig. 2.

To address the concern of increased heating rates over dielectric materials [17], we shuttle our ion from the loading hole, where it is above bare aluminum electrodes, to a location directly over the output grating of a waveguide. Here, the ion is in a direct line of sight with the

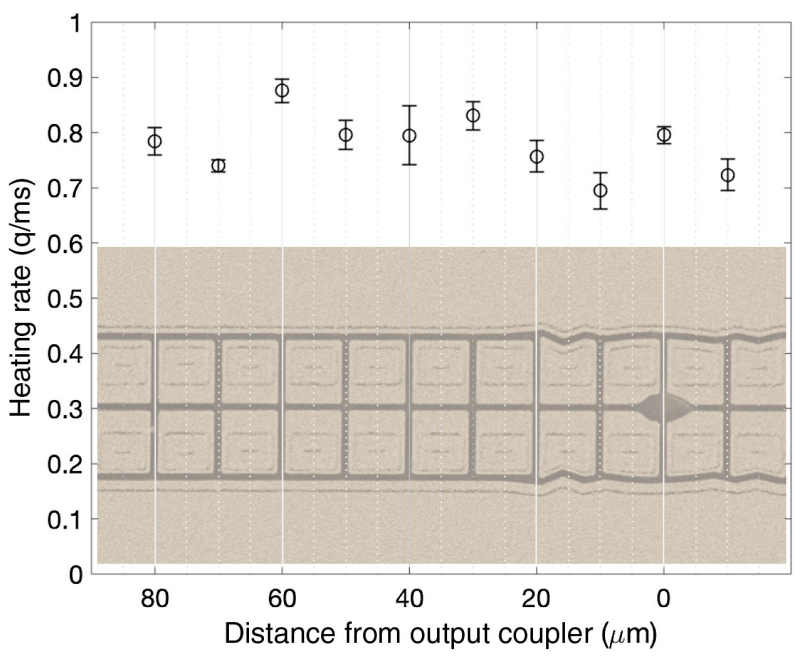

FIG. 3. Heating rate as a function of position overlayed on the trap region, where the output coupler for the waveguide is at $0 \mu \mathrm{m}$, the loading hole is at $80 \mu \mathrm{m}$, and the maximum overlap between the ion and the waveguide output light is at $70 \mu \mathrm{m}$. No measurable change in heating rate is observed as the ion is shuttled from the loading hole to the output grating where it is closest to the exposed dielectric material. 


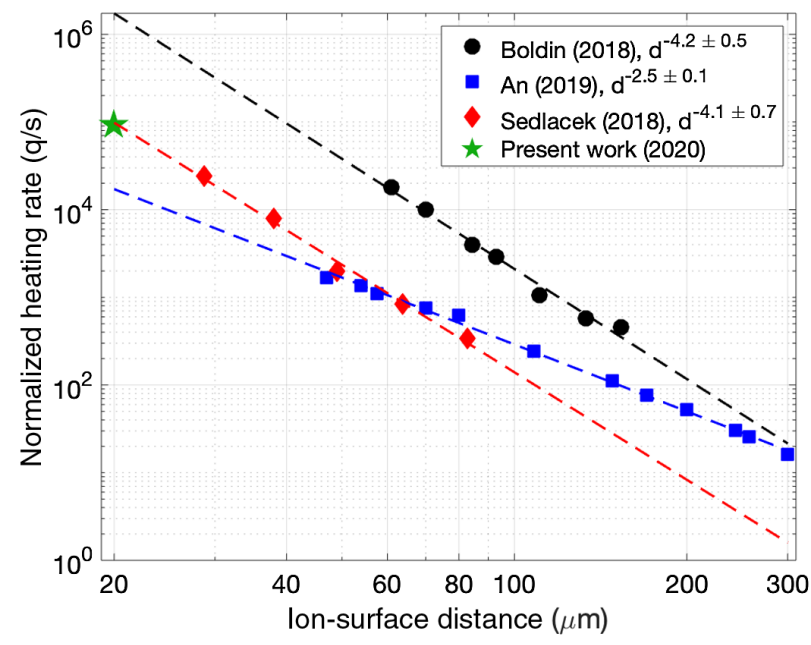

FIG. 4. Heating rate as a function of the ion-surface distance. The heating rate has been normalized for ion mass and secular motional trap frequency. We plot our data as the green star compared to previously published data from Refs. [14] (black circles), [24] (blue squares), and [15] (red diamonds). The scaling of each fit is indicated in the legend. Data are reprinted with permission from the authors of Ref. [24].

silicon nitride waveguide. Figure 3 shows the heating rate at $10-\mu \mathrm{m}$ increments over a range of $80 \mu \mathrm{m}$ between the loading hole and the dielectric output grating. Remarkably, there is no measurable change in heating rate even when the ion is positioned directly above the dielectric output grating at a height of $20 \mu \mathrm{m}$. The mean heating rate across the chip is $0.78 \pm 0.05 \mathrm{q} / \mathrm{ms}$.

We can normalize our measured heating rate for ion mass and trap frequency to compare with community data $[16,23,24]$. To do so, we consider the heating rate given by $\langle\dot{n}\rangle=\left(q^{2} / 4 m \hbar \omega\right) S_{E}$, where $q$ is the charge of the ion, $m$ is the mass of the ion, $\omega$ is the motional frequency, and $S_{E}$ is

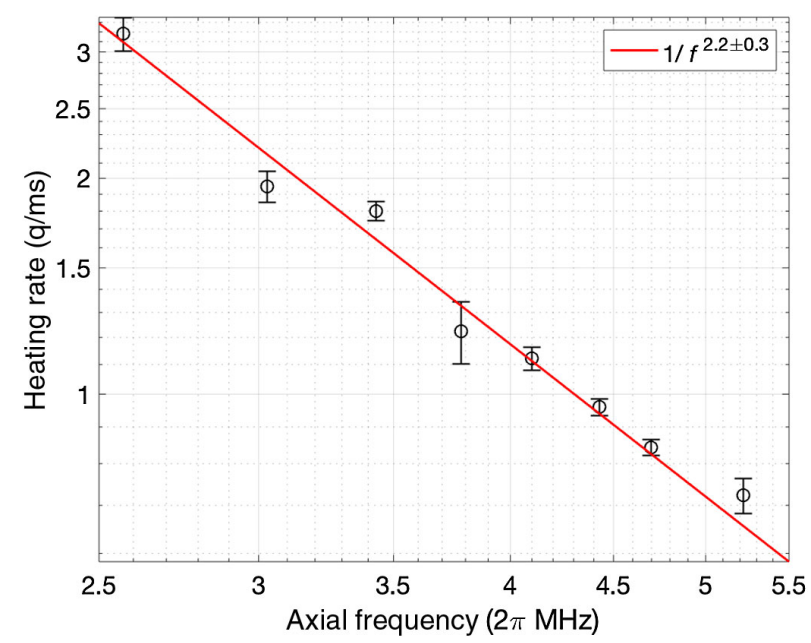

FIG. 5. Heating rate as a function of axial secular motional trap frequency following a $1 / f^{2.2 \pm 0.3}$ trend consistent with $1 / f$ scaling of electric field spectral density $[15,24]$. the spectral density of the electric field noise, which goes like $1 / \omega$. Scaling to $\omega=2 \pi \times 1 \mathrm{MHz}$ and ${ }^{40} \mathrm{Ca}^{+}$allows us to compare our data point at a $20-\mu \mathrm{m}$ ion-surface distance with published data at larger ion-surface distances. Figure 4 shows that our normalized heating rate falls along the extrapolated $d^{-4}$ scaling found in other experiments $[14,15,24]$.

We also measure the heating rate as a function of axial secular trap frequency over the loading hole. Figure 5 shows our results as we scan the frequency from $2 \pi \times 2.5$ to $2 \pi \times 5.2 \mathrm{MHz}$. The fitted line represents a power law $\langle\dot{n}\rangle \propto 1 / f^{2.2 \pm 0.3}$, which agrees well with previously reported heating rate trends and is consistent with $1 / f$ scaling of electric field spectral density $[15,24]$.

\section{B. Waveguide beam profile}

We couple 435-nm light into the waveguide via the input diffraction grating shown in Fig. 1(d), which emits light from the output grating at a measured angle of $66^{\circ}$ [Fig. 1(f)]. This differs from the simulated output angle of $63^{\circ}$ due to fabrication tolerances. Roughly $26 \mathrm{~mW}$ of power is incident on the input grating. We determine the output intensity by measuring the Rabi rate of the ion at various positions around the expected grating output and plot the relative Rabi frequencies as a function of ion position for the $\Delta m_{F}=+1$ transition (see Fig. 6). In this device, we observed a double-peak profile from the grating. Note the dip in transmission near $11 \mu \mathrm{m}$ from the output grating. We attribute this to destructive interference in the

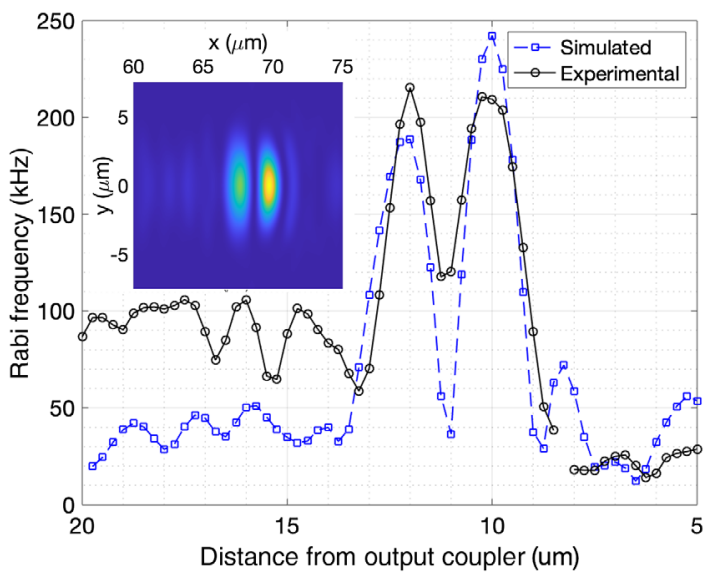

FIG. 6. Measured (black) and simulated (blue) Rabi frequency of the $\Delta m_{F}=+1$ transition using the waveguide-delivered beam as a function of the ion's distance from the loading hole. The two maxima, indicating highest beam intensities, occur when the ion is $10 \mu \mathrm{m}$ and $12 \mu \mathrm{m}$ from the output grating. The dip is attributed to variations in the distance between the grating and reflective layer and can be reproduced in simulations that vary this distance by $60 \mathrm{~nm}$ (blue). The simulated Rabi frequency is calculated from the simulated waveguide output intensity and is scaled to match the experimental Rabi frequency. Inset: simulated 2D waveguideoutput-intensity cross section at the ion height. 
output beam due to variations in the distance between the grating and reflector described in Sec. II. Because of limitations in the fabrication tolerances, there are variations in the oxide thickness across the wafer, and therefore, the actual distance between the grating and reflector varies across the wafer. This variation in spacing between the grating and reflective surface is a result of the chemical mechanical polishing process that is used to polish the oxide layer above the metal, prior to deposition of the silicon nitride waveguide layer. This process results in variation of the oxide thickness across the wafer on the order of $100 \mathrm{~nm}$.

This double-peak feature was reproduced in simulation by vertically shifting the location of the reflector by $60 \mathrm{~nm}$ from the nominal design point, which results in a reduction of the output efficiency from $-1.6 \mathrm{~dB}$ in the ideal case to $-3.7 \mathrm{~dB}$, and two submicron peaks separated by $1.8 \mu \mathrm{m}$. The simulated output intensity profile with this $60-\mathrm{nm}$ shift is shown as a $2 \mathrm{D}$ cross section at the anticipated ion height in the inset of Fig. 6. The simulated 1D Rabi frequency corresponding to $y=0 \mu \mathrm{m}$ in the inset is shown in blue in Fig. 6.

In future devices, the fabrication uncertainty will be reduced by altering the fabrication process such that the thickness of the oxide between the metal reflector and grating are set by a single deposition process, without a polishing step. This approach is expected to reduce crosswafer oxide variation below $10 \mathrm{~nm}$ and eliminate the double peak in the output profile.

Despite this feature, we have observed $\pi$ times as fast as $4.13 \pm 0.02 \mu$ s corresponding to Rabi frequencies as high as $\Omega=2 \pi \times 121.1 \pm 0.6 \mathrm{kHz}$ when coupling to the waveguide is optimized. This case corresponds to an estimated peak intensity of nearly $300 \mathrm{nW} / \mu \mathrm{m}^{2}$ and through-chip coupling of $-39 \mathrm{~dB}$.

\section{Electrostatic charging measurements and results}

In some traps with integrated photonics, an ITO coating is deposited over the exposed dielectrics to prevent charge buildup [12]. For our traps, ITO was not employed as it was not compatible with the CMOS facility used at Sandia. Consequently, we observe an effect due to photoliberated electrons charging the $35-\mu \mathrm{m}^{2}$ area of exposed dielectric material (corresponding to a 0.31 -steradian solid angle) that composes the output grating coupler of the integrated waveguide. The electric field from the charged grating coupler simultaneously shifts the position of the ion and perturbs the harmonic potential of the prescribed trapping fields. We measure this effect by monitoring the axial motional sideband frequency in two different scenarios: (1) Charging is induced via continuously applied light through the waveguide (off-resonant), and the sideband frequency is probed via a free-space beam; (2) charging is induced and the sideband frequency is probed via light coupled through the waveguide. In the latter case, the light through the waveguide is pulsed on only during the probe time, which is more representative of normal operation.

\section{Continuous photoinduced charging}

We first investigate the photoinduced charging effect at a long timescale by measuring the axial secular frequency with a free-space beam. Using a free-space beam to probe the ion allows us to maintain a constant optical power through the waveguide over the duration of the measurement. The secular frequency is measured from the motional sideband of the $\left|2 S_{1 / 2}, F=0, m_{F}=0\right\rangle$ to $\mid 2 D_{3 / 2}, F=$ $\left.2, m_{F}=+2\right\rangle$ transition. This measurement is performed with the ion directly above the grating ( $80 \mu \mathrm{m}$ from the loading hole in Fig. 6). We begin with the waveguidecoupled light shuttered. We interrogate the resonant frequencies of the red and blue axial sidebands approximately every $15 \mathrm{~s}$ while the waveguide coupler is continuously charged for $2000 \mathrm{~s}$ by turning on the nonresonant waveguide-coupled light and then discharged for $2500 \mathrm{~s}$ by turning off the waveguide-coupled light.

The results of the charging experiment are shown in Fig. 7. The first $400 \mathrm{~s}$ shows the baseline axial frequency after the waveguide has been off for a period of 12 hours. We turn on the waveguide-coupled light at $400 \mathrm{~s} f$. The charging is characterized by two main effects. The primary effect is a fast negative charging of the top metal layer, which is attributed to a small electric dipole created when electrons are excited from the metal onto insulating patches of oxide above [25]. This effect is superimposed by a

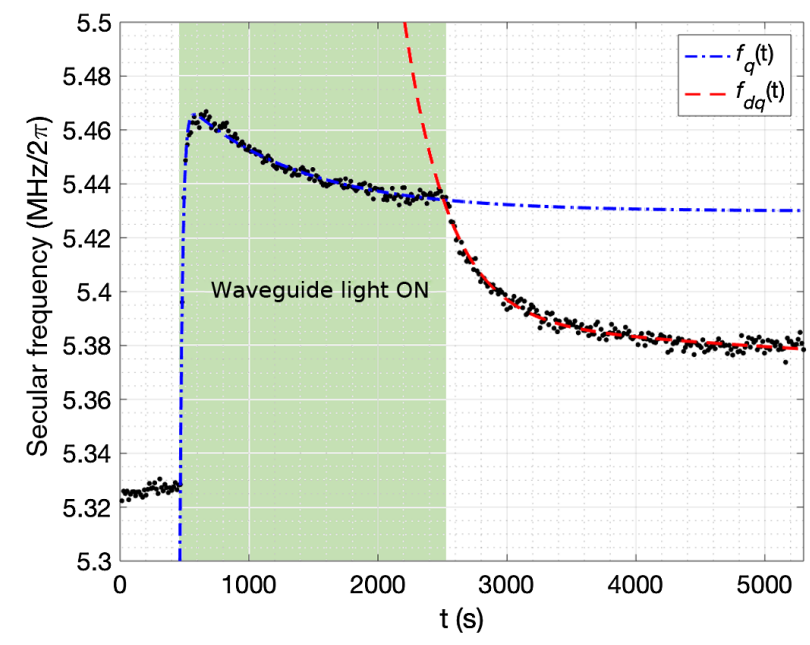

FIG. 7. Secular frequency vs time as waveguide-coupled light is turned on and off. Secular frequency is measured with a freespace beam tuned to the $\Delta m_{F}=+2$ transition. Between $500 \mathrm{~s}$ and $2500 \mathrm{~s}$, the waveguide-coupled light is turned on (green) and servoed to a constant power. The two opposing charge effects are attributed to the metal and dielectric layers as described in Ref. [25]. Fitted lines for the charging (blue dot dashed) and discharging (red dashed) are described by Eqs. (1) and (2). 
slower secondary effect of positive charging of the dielectric grating. These two effects have previously been observed and are well characterized in similar photoinduced charge studies [25]. When the waveguide-coupled light is turned off at $2500 \mathrm{~s}$, both materials slowly discharge.

With the light on, the frequency shift due to charging $f_{q}(t)$ can be fit to the following form:

$$
\begin{aligned}
f_{q}(t)= & \Delta f_{1}\left[1-\exp \left(\frac{-\left(t-t_{\mathrm{on}}\right)}{T_{1}}\right)\right] \\
& -\Delta f_{2}\left[1-\exp \left(\frac{-\left(t-t_{\mathrm{on}}\right)}{T_{2}}\right)\right]+f_{0},
\end{aligned}
$$

where $\Delta f_{1,2}$ are the settling frequency offsets of the two charging effects. The difference $\Delta f_{1}-\Delta f_{2}$ represents the settling frequency offset with the waveguide light on. The terms $t_{\mathrm{on}}$ and $T_{1,2}$ represent the turn-on time of the waveguide light and the charging time, respectively. The initial frequency prior to turning on the waveguide light is $f_{0}$.

After the waveguide light has been extinguished, the frequency shift due to discharging $f_{d q}(t)$ can be described as follows:

$$
\begin{aligned}
f_{d q}(t)= & \Delta f_{3}\left[-\exp \left(\frac{-\left(t-t_{\text {off }}\right)}{T_{3}}\right)\right] \\
& +\Delta f_{4}\left[-\exp \left(\frac{-\left(t-t_{\text {off }}\right)}{T_{4}}\right)\right]+f_{0},
\end{aligned}
$$

where $\Delta f_{3,4}$ and $T_{3,4}$ are the settling frequency offsets and the discharging times of the two materials, respectively, and $t_{\text {off }}$ is the turn-off time of the waveguide light.

In Fig. 7, upon turning on the waveguide, the charging happens over $T_{1}=21 \mathrm{~s}$ and $T_{2} \sim 900 \mathrm{~s}$, and the difference of the settling frequency offsets is $\Delta f_{1}-\Delta f_{2}=101 \mathrm{kHz}$. After the waveguide turns off, the discharging times are $T_{3} \sim 6 \mathrm{~min}$ and $T_{4} \sim 5 \mathrm{hrs}$ as the frequency is expected to return to its original $f_{0}$ value of $5.329 \mathrm{MHz}$. We can negate the settled frequency offset $\Delta f_{1}-\Delta f_{2} \simeq 0.1 \mathrm{MHz}$ by applying an electric field of about $2.4 \mathrm{kV} / \mathrm{cm}$ in the vertical direction.

For this experiment, approximately $17 \mathrm{~mW}$ of offresonant $435-\mathrm{nm}$ light is directed on the input coupler to the waveguide. To better understand how much power is coupled through the waveguide, we direct the same amount of power of resonant 435-nm light onto the input coupler, and we shuttle the ion $68 \mu \mathrm{m}$ from the loading hole (see Fig. 6) where the waveguide-outcoupled light is maximally overlapped with the ion. Similar to the beam profile experiments in Sec. III B, we use the waveguide-coupled light to perform Rabi flops on the $\left|2 S_{1 / 2}, F=0, m_{F}=0\right\rangle$ to $\left|2 D_{3 / 2}, F=2, m_{F}=+1\right\rangle$ transition and achieve $\pi$ times of $5 \mu \mathrm{s}$ for this particular experiment. Because of the angle of the waveguide beam with respect to our magnetic field,

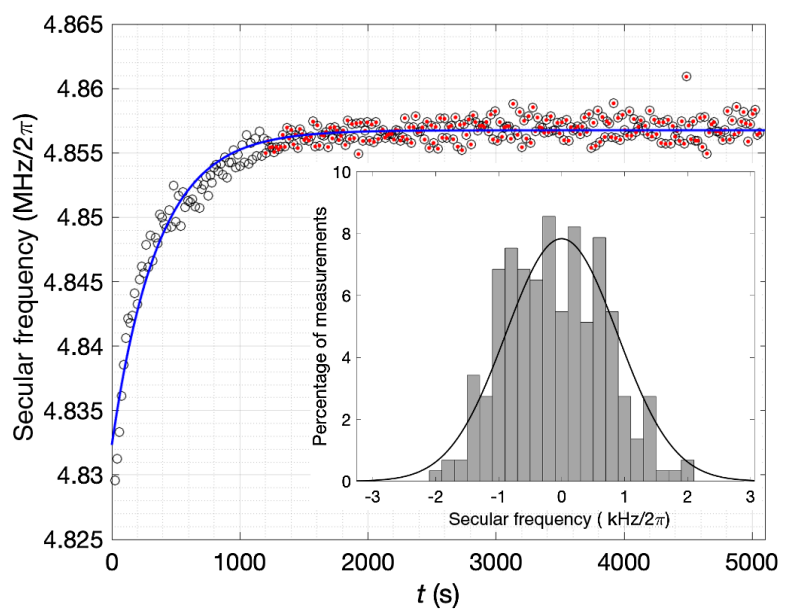

FIG. 8. Secular frequency over time during typical clock operation, as measured by the waveguide-coupled light tuned to the $\Delta m_{F}=1$ transition. Inset: histogram of the settled frequency (red points) difference from the fitted curve (blue line). The histogram is fitted to a Gaussian with $\sigma=0.9 \mathrm{kHz}$.

the $\Delta m_{F}=+1$ transition is most strongly coupled by the waveguide beam.

\section{Charging due to pulsed operation}

We also monitor the charge-induced frequency shifts using the waveguide-coupled light rather than the freespace light in order to determine the stability of the shifted frequency during more typical clock operation. Figure 8 shows the frequency shift of the $\Delta m_{F}=1$ transition during a typical duty cycle. The charging happens on a much slower timescale because the light is only on for a fraction of the time as shown in the measurements in Fig. 7. In these experiments, the waveguide-coupled light is only on during the probe time for $15 \mu \mathrm{s}$, which contributes $0.61 \%$ of the duty cycle. A histogram of the difference between the fitted exponential and the data points is shown in the inset with a standard deviation of $\sigma=0.9 \mathrm{kHz}$.

\section{CONCLUSION}

We have fabricated a surface trap with integrated waveguides for delivering 435-nm light to address the quadrupole transition in ${ }^{171} \mathrm{Yb}^{+}$. To assess the feasibility of dielectric waveguides in quantum applications, we have characterized the heating rate as a function of proximity to exposed dielectric output grating and of axial secular trap frequency. We see no increase in the heating rate over the dielectric, despite having an ion-surface distance of only $20 \mu \mathrm{m}$. The heating rate scaling with axial secular trap frequency follows a $1 / f^{2.2}$ trend, consistent with previous results.

We also mapped the output profile of the waveguide by measuring the Rabi frequency of the ion with the waveguide-delivered light and showed that it is consistent with 
simulations and trap fabrication tolerances. We anticipate that the beam profile will significantly improve in future devices by eliminating the double-peak feature present in the current device output. Furthermore, transitioning to an edge-coupled instead of diffraction grating-coupled device at the input is anticipated to improve the input coupling to $-3 \mathrm{~dB}$ or better, depending on the process used. Despite this feature, we have measured $\pi$ times as fast as $4.13 \pm$ $0.02 \mu \mathrm{s}$.

Finally, we have characterized the electric field shift and stability due to the photoinduced charging of the trap. The lack of deleterious heating over exposed dielectrics or destabilizing photoinduced electric fields is a promising result for the future utilization of these devices in quantum computing and timekeeping applications.

\section{ACKNOWLEDGMENTS}

The authors thank the members of Sandia's Microsystems and Engineering Sciences Applications (MESA) facility for their fabrication expertise and for helpful comments on the manuscript, as well as H. Haeffner and D. An for their discussions and data for Fig. 4. This work was supported by the Defense Advanced Research Projects Activity (DARPA). Sandia National Laboratories is a multimission laboratory managed and operated by National Technology \& Engineering Solutions of Sandia, LLC, a wholly owned subsidiary of Honeywell International Inc., for the U.S. Department of Energy's National Nuclear Security Administration under Contract No. DENA0003525. This paper describes objective technical results and analyses. Any subjective views or opinions that might be expressed in the paper do not necessarily represent the views of the U.S. Department of Energy or the United States Government.

[1] D. Stick, W. K. Hensinger, S. Olmschenk, M. Madsen, K. Schwab, and C. Monroe, Ion Trap in a Semiconductor Chip, Nat. Phys. 2, 36 (2006).

[2] S. Seidelin, J. Chiaverini, R. Reichle, J. J. Bollinger, D. Leibfried, J. Britton, J. H. Wesenberg, R. B. Blakestad, R. J. Epstein, D. B. Hume, W. M. Itano, J. D. Jost, C. Langer, R. Ozeri, N. Shiga, and D. J. Wineland, Microfabricated Surface-Electrode Ion Trap for Scalable Quantum Information Processing, Phys. Rev. Lett. 96, 253003 (2006).

[3] S. M. Brewer, J.-S. Chen, A. M. Hankin, E. R. Clements, C. W. Chou, D. J. Wineland, D. B. Hume, and D. R. Leibrandt, ${ }^{27} \mathrm{Al}^{+}$Quantum-Logic Clock with a Systematic Uncertainty below 10 ${ }^{-18}$, Phys. Rev. Lett. 123, 033201 (2019).

[4] S. M. Brewer, J.-S. Chen, K. Beloy, A. M. Hankin, E. R. Clements, C. W. Chou, W. F. McGrew, X. Zhang, R. J. Fasano, D. Nicolodi, H. Leopardi, T. M. Fortier, S. A. Diddams, A. D. Ludlow, D. J. Wineland, D. R. Leibrandt, and D. B. Hume, Measurements of ${ }^{27} \mathrm{Al}^{+}$and ${ }^{25} \mathrm{Mg}^{+}$
Magnetic Constants for Improved Ion-Clock Accuracy, Phys. Rev. A 100, 013409 (2019).

[5] J. Borregaard and A.S. Sorensen, Efficient Atomic Clocks Operated with Several Atomic Ensembles, Phys. Rev. Lett. 111, 090802 (2013).

[6] K. Mehta, C. Bruzewicz, R. McConnell, R. J. Ram, J. M. Sage, and J. Chiaverini, Integrated Optical Addressing of an Ion Qubit, Nat. Nanotechnol. 11, 1066 (2016).

[7] L. Jiang, W. B. Whitten, and S. Pau, A Planar Ion Trapping Microdevice with Integrated Waveguides for Optical Detection, Opt. Express 19, 3037 (2011).

[8] D. H. Slichter, V. B. Verma, D. Leibfried, R. P. Mirin, S. W. Nam, and D. J. Wineland, UV-Sensitive Superconducting Nanowire Single Photon Detectors for Integration in an Ion Trap, Opt. Express 25, 8705 (2017).

[9] K. Mehta, C. Zhang, M. Malinowski, T.-L. Nguyen, M. Stadler, and J.P. Home, Integrated Optical Multi-Ion Quantum Logic, Nature (London) 586, 533 (2020).

[10] G. N. West, W. Loh, D. Kharas, C. SoraceAgaskar, K. K. Mehta, J. Sage, J. Chiaverini, and R. J. Ram, Low-Loss Integrated Photonics for the Blue and Ultraviolet Regime, APL Photonics 4, 026101 (2019).

[11] C. Sorace-Agaskar, D. Kharas, S. Yegnanarayanan, R. T. Maxson, G. N. West, W. Loh, S. Bramhavar, R. J. Ram, J. Chiaverini, J. Sage, and P. Juodawlkis, Versatile Silicon Nitride and Alumina Integrated Photonic Platforms for the Ultraviolet to Short-Wave Infrared, IEEE J. Sel. Top. Quantum Electron. 25, 8201515 (2019).

[12] R. J. Niffenegger, J. Stuart, C. Sorace-Agaskar, D. Kharas, S. Bramhavar, C. D. Bruzewicz, W. Loh, R. McConnell, D. Reens, G. N. West, J. M. Sage, and J. Chiaverini, Integrated Multi-Wavelength Control of an Ion Qubit, Nature (London) 586, 538 (2020).

[13] M. Brownnutt, M. Kumph, P. Rabl, and R. Blatt, Ion-Trap Measurements of Electric-Field Noise Near Surfaces, Rev. Mod. Phys. 87, 1419 (2015).

[14] I. A. Boldin, A. Kraft, and C. Wunderlich, Measuring Anomalous Heating in a Planar Ion Trap with Variable Ion-Surface Separation, Phys. Rev. Lett. 120, 023201 (2018).

[15] J. A. Sedlacek, A. Greene, J. Stuart, R. McConnell, C. D. Bruzewicz, J. M. Sage, and J. Chiaverini, Distance Scaling of Electric-Field Noise in a Surface-Electrode Ion Trap, Phys. Rev. A 97, 020302(R) (2018).

[16] D. A. Hite, Y. Colombe, A. C. Wilson, K. R. Brown, U. Warring, R. Jördens, J. D. Jost, K. S. McKay, D. P. Pappas, D. Leibfried, and D. J. Wineland, 100-Fold Reduction of Electric-Field Noise in an Ion Trap Cleaned with In Situ Argon-Ion-Beam Bombardment, Phys. Rev. Lett. 109, 103001 (2012).

[17] M. Kumph, C. Henkel, P. Rabl, M. Brownnutt, and R. Blatt, Electric-Field Noise above a Thin Dielectric Layer on Metal Electrodes, New J. Phys. 18, 023020 (2016).

[18] K. R. Brown, J. Kim, and C. Monroe, Co-Designing a Scalable Quantum Computer with Trapped Atomic Ions, njp Quantum Inf. 2, 16034 (2016).

[19] J. M. Pino, J. M. Dreiling, C. Figgatt, J. P. Gaebler, S. A. Moses, C. H. Baldwin, M. Foss-Feig, D. Hayes, K. Mayer, C. Ryan-Anderson, and B. Neyenhuis, Demonstration of the 
QCCD Trapped-Ion Quantum Computer Architecture, Nature (London) 592, 209 (2021).

[20] D. L. Moehring, C. Highstrete, D. Stick, K. M. Fortier, R. Haltli, C. Tigges, and M. G. Blain, Design, Fabrication and Experimental Demonstration of Junction Surface Ion Traps, New J. Phys. 13, 075018 (2011).

[21] M. Johanning, A. Braun, D. Eiteneuer, C. Paape, C. Balzer, W. Neuhauser, and C. Wunderlich, Resonance-Enhanced Isotope-Selective Photoionization of YbI for Ion Trap Loading, Appl. Phys. B 103, 327 (2011).

[22] C. F. Roos, Ph.D. thesis, University of Innsbruck (2000).
[23] C. Noel, M. Berlin-Udi, C. Matthiesen, J. Yu, Y. Zhou, V. Lordi, and H. Haffner, Electric-Field Noise from Thermally Activated Fluctuators in a Surface Ion Trap, Phys. Rev. A 99, 063427 (2019).

[24] D. An, C. Matthiesen, E. Urban, and H. Haffner, Distance Scaling and Polarization of Electric-Field Noise in a Surface Ion Trap, Phys. Rev. A 100, 063405 (2019).

[25] M. Harlander, M. Brownnutt, W. Hänsel, and R. Blatt, Trapped-Ion Probing of Light Induced Charging Effects on Dielectrics, New J. Phys. 12, 093035 (2012). 